\title{
Perfil das indústrias moveleiras da região Metropolitana do Rio de Janeiro
}

\author{
Natália Dias de Souza ${ }^{1}$, Alexandre Monteiro de Carvalho ${ }^{1}$, Eduardo Vinicius da Silva ${ }^{1}$, \\ Fábio Minoru Yamaji ${ }^{2}$, Ananias Francisco Dias Júnior ${ }^{3 *}$ \\ ${ }^{1}$ Departamento de Produtos Florestais, Universidade Federal Rural do Rio de Janeiro, Rodovia BR 465, Km 07 - Zona Rural - \\ CEP 23890-000, Seropédica - RJ, Brasil. \\ ${ }^{2}$ Universidade Federal de São Carlos, Campus Sorocaba, Grupo de Biomassa e Bionergia. Rodovia João Leme dos Santos, \\ (SP-264), Km 110, s/n - Itinga, CEP 18052-780, Sorocaba - SP, Brasil. \\ 3 Doutorando em Recursos Florestais, Universidade de São Paulo, Escola Superior de Agricultura "Luiz de Queiroz", \\ Av. Pádua Dias, 11 - Agronomia - CEP 13418-900, Piracicaba - SP, Brasil.
}

\begin{abstract}
RESUMO Este trabalho teve por objetivo analisar o perfil da atividade moveleira da região metropolitana do Estado do Rio de Janeiro. O estudo utilizou a aplicação de questionários pelo setor produtivo de cada empresa avaliada. A produção moveleira pôde ser dividida em duas classes, sendo uma representada pela maioria dos produtores moveleiros (70\%), composta principalmente por pequenos produtores dedicados à fabricação de móveis, utilizando poucas máquinas, com a produção por encomenda e ausência de controle de qualidade. A segunda classe é representada por fábricas modernas, contendo parque industrial, representada por empresas familiares e de capital nacional. A produção é representada por móveis sob medida, com emprego de MDP (Medium Density Particleboard) e MDF (Medium Density Fiberboard). O foco é em móveis residenciais e a comercialização é realizada através do contato direto entre consumidor/fábrica. As questões socioambientais estão incorporadas no contexto produtivo das empresas.
\end{abstract}

Palavras-chave: setor moveleiro; produção e desenvolvimento; gestão pela qualidade.

\section{Profile of the furniture companies from the Metropolitan region of Rio de Janeiro}

\begin{abstract}
This study aimed to analyze the profile of the furniture production in the metropolitan region of the state of Rio de Janeiro. The study was based on questionnaires to the productive sector of each evaluated company. The furniture production could be divided into two classes, in which one of them is represented by the majority of the furniture companies (70\%), composed mainly by small manufacturers, using few machines and producing by demand and with a lack of quality control. The second class is represented by modern companies containing industrial park, represented by family companies with national capital. Their production is represented by custom-made furniture, using MDP (Medium Density Particleboard) and MDF (Medium Density Fiberboard). The focus is on residential furniture and the commercialization is carry out directly with the final client. The social and environmental issues are incorporated into the productive context of the companies.
\end{abstract}

Keywords: furniture company; production and development; quality by management.

\section{Introdução}

Dentro da indústria de transformação, a fabricação de móveis, em especial os de madeira, pode ser considerada uma das atividades mais tradicionais. O setor moveleiro reúne características específicas, como grande utilização de insumos de origem natural, intensivo uso da força de trabalho e elevado grau de informalidade. $\mathrm{O}$ setor ainda pode ser dividido 
em segmentos de acordo com sua utilidade (móveis institucionais, residenciais e de escritório); material predominante para sua confecção (madeira e derivados, plásticos e metais); classe de consumo para a qual é projetado (A, B, C, D ou E) e até mesmo a faixa etária dos prováveis consumidores (GALINARI et al., 2013).

De acordo com Cassilha et al. (2004), a retração econômica ocorrida na década de 80 proporcionou cortes de investimentos, trazendo uma crescente desatualização do setor moveleiro diante do cenário mundial, que introduzia nessa mesma época a microeletrônica como parte integrante e revolucionária no setor de máquinas e equipamentos.

A indústria brasileira de fabricação de móveis é formada por 18.200 estabelecimentos, que empregam aproximadamente 330 mil profissionais em todo país. Em 2013, essas empresas produziram 511,8 milhões de peças para o mercado nacional que significam $\mathrm{R} \$ 42,9$ bilhões (EXATA COMUNICAÇÃO, 2014).

As principais empresas localizam-se nas regiões sudeste e sul do país. Os polos moveleiros que mais se destacam no cenário nacional estão localizados nos Estados de Santa Catarina, São Paulo, Minas Gerais, Paraná e Rio Grande do Sul. Salienta-se ainda o surgimento de importantes polos nas regiões Centro-Oeste, Norte e Nordeste, considerados "emergentes" visto que nos próximos anos poderão desempenhar papel importante no cenário nacional e internacional da indústria moveleira (ABDI, 2009b).

Nos últimos anos, a população brasileira tem adquirido uma maior quantidade de móveis devido ao aumento da renda, do número de empregos, da maior facilidade de acesso ao crédito e dos incentivos ao consumo, tal como o oferecido pelo programa "Minha Casa Melhor" (GALLUCCI, 2014). A indústria do mobiliário nacional permanecerá com o mercado doméstico cativo no futuro imediato e será necessário desenvolver ações internas para obtenção de vantagens competitivas dinâmicas e impedir a entrada de concorrentes externos (ABDI, 2009a).

Os principais centros consumidores são as regiões Sul e Sudeste, com destaque para São Paulo e região do ABC, Porto Alegre, Curitiba, Belo Horizonte, Rio de Janeiro e Brasília, bem como suas respectivas regiões metropolitanas (ROSA et al., 2007).

O Rio de Janeiro, segundo maior mercado consumidor de móveis do Brasil, começou a sua produção em 1890 com a inauguração da Companhia de Móveis Curvados (AMBROS, 2011). Ao longo das últimas décadas, o estado só perdeu posição de produção devido, principalmente, às práticas de políticas tributárias extremamente agressivas.

Dentro do estado do Rio de Janeiro existem aproximadamente 580 estabelecimentos destinados à fabricação de móveis, os quais geram 7.509 empregos (SANT ANNA, 2013). Esses produtores não conseguem sustentar o mercado, devido a demanda e ao crescimento econômico e populacional da região. Grande parte dos móveis consumidos no Rio de Janeiro é obtida de outra região. Exemplo disso é o polo moveleiro de Ubá-MG que contribui com aproximadamente 50\% da produção para o Rio de Janeiro, metrópole mais próxima do município (SANT ANNA, 2013).

O setor de móveis fluminense irá trabalhar nos próximos anos em parceria com o Sistema da Fundação das Indústrias do Estado do Rio de Janeiro (FIRJAN) em um plano de metas e iniciativas prioritárias com o objetivo de atualizar as informações sobre o segmento e de criar estratégias para incentivar o crescimento da base sindical (FIRJAN, 2013b).

Diante do exposto, o presente trabalho teve por objetivo levantar o perfil da atividade moveleira da região metropolitana do Estado do Rio de Janeiro com base nos aspectos tecnológicos e produtivos das indústrias. 


\section{Material e Métodos}

A área metropolitana foi escolhida pelo fato de que 57\% das indústrias moveleiras do Estado do Rio de Janeiro situamse nessa região (SANT ANNA, 2013), como apresentado na

Figura 1.

Foram identificados dois sindicatos ligados às indústrias de móveis vigentes da região (Sindicato das Indústrias de Móveis de Madeira do Município do Rio de Janeiro - SIM-Rio e Sindicato das Indústrias de Construção Civil e do Mobiliário da Baixada Fluminense - SINCOCIMO). Por meio dos sindicatos, obteve-se o número total de empresas moveleiras da região.

As visitas ocorreram entre os meses de janeiro e julho do ano de 2015. Dessa maneira, aplicaram-se questionários ao responsável do setor produtivo de cada empresa, contendo perguntas de informações relacionadas aos aspectos tecnológicos, tais como: desenvolvimento e inovação tecnológica; frequência de alterações no desenho ou projeto dos móveis; definição de layout; dificuldades em obtenção da matériaprima e matérias-primas utilizadas pela empresa no sistema produtivo. As respostas dos questionários foram analisadas por meio de estatística descritiva e sistematizadas por meio de tabulação.

\section{Resultados e Discussão}

Foram encontrados trinta estabelecimentos dedicados à fabricação de móveis. Pode-se verificar que 70\% desses estabelecimentos são pequenos espaços físicos, sem layout definido e sem planta industrial, que utilizam métodos de produção e equipamentos desatualizados, além da ausência de gestão definida.

Porém, dentro desse universo de indústrias filiadas, encontraram-se nove empresas com características peculiares, ou seja, fábricas estruturadas, com parque industrial, empresas com nicho de mercado específico que produzem uma diversidade de produtos, com lojas revendedoras e sítios estruturados.

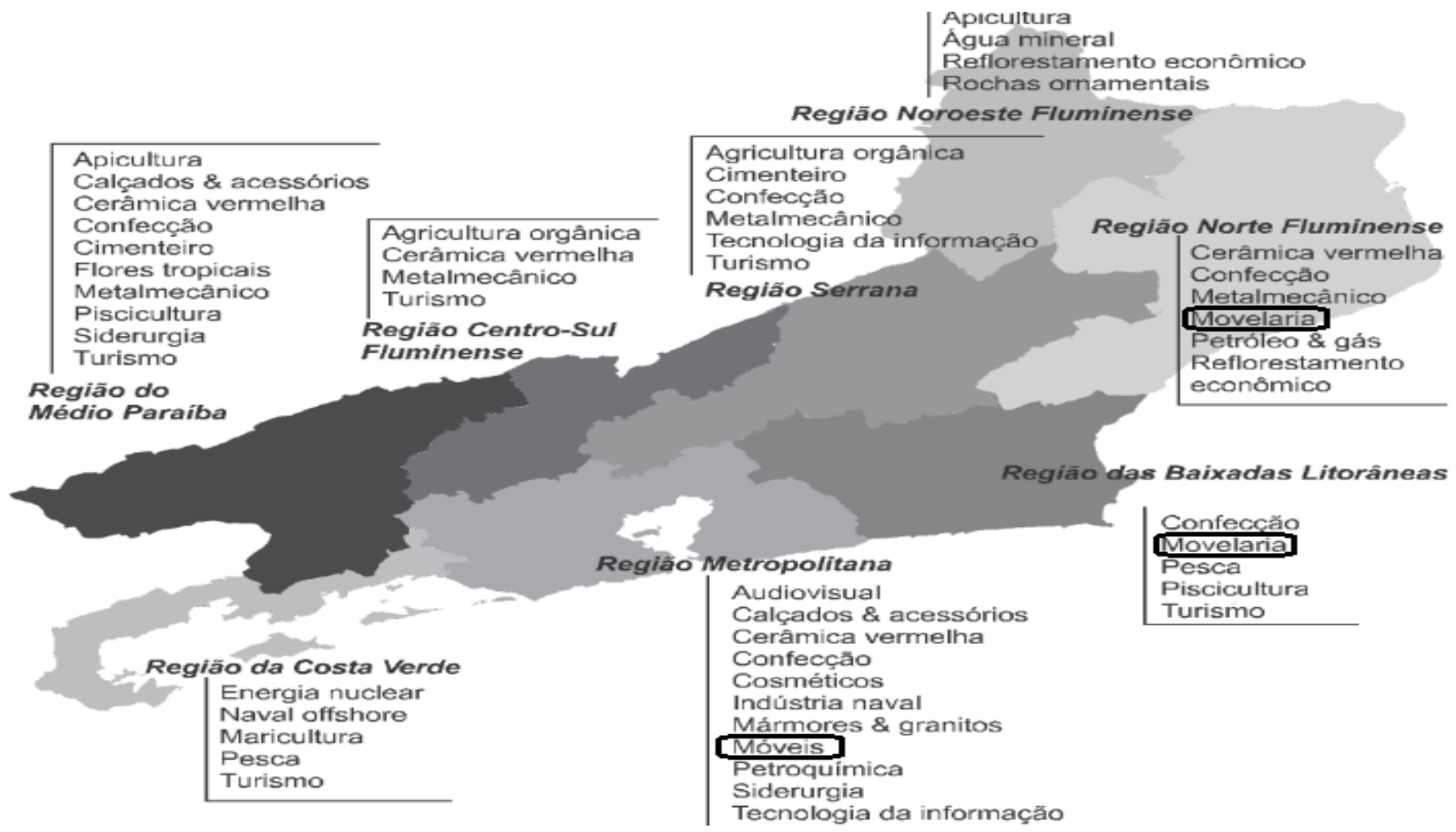

Figura 1. Mapa das distribuições econômicas do estado do Rio de Janeiro. (Fonte: SANT ANNA, 2013).

Figure 1. Map of the economic distributions of Rio de Janeiro State (Source: SANT ANNA, 2013). 
Os resultados a seguir referem-se a essas empresas com parque fabril. Vale ressaltar que do total de indústrias (nove), cinco responderam à pesquisa.

\section{Perfil das empresas}

Para a classificação das empresas utilizou-se a definição do Serviço Brasileiro de Apoio às Micro e Pequenas Empresas (SEBRAE, 2013) que classifica o porte das indústrias pelo número de funcionários. De acordo com as características presentes na Figura 2, as empresas moveleiras investigadas foram classificadas como de pequeno porte.

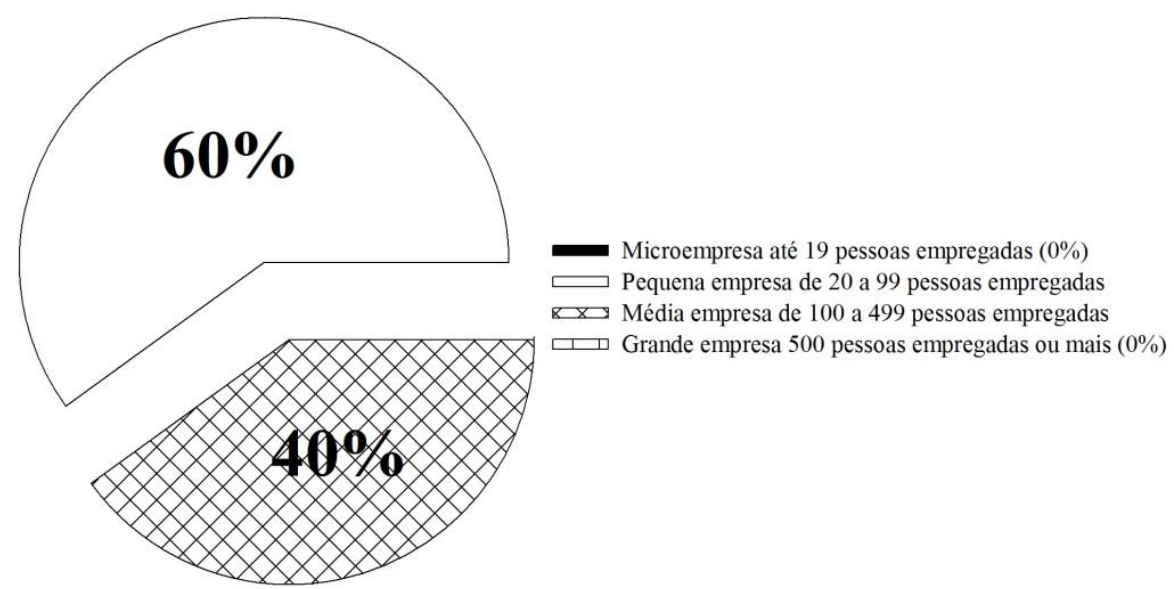

Figura 2. Classificação das empresas moveleiras de acordo com o número de funcionários.

Figure 2. Classification of furniture companies according to the number of employees.

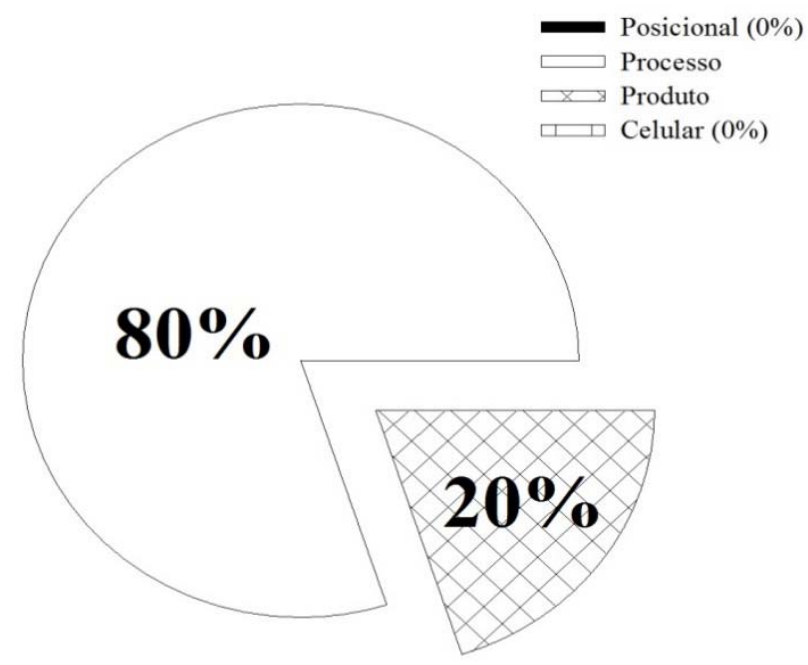

Figura 3. Layout utilizado pelas indústrias moveleiras da região metropolitana do Rio de Janeiro.

Figure 3. Layout used by the furniture companies in the metropolitan region of Rio de Janeiro. 
indústrias moveleiras internacionais, que são constituídas na sua maioria de pequenas e médias empresas (ARRUDA, 2009).

As empresas estudadas possuem em média 40 anos de existência, sendo que há empresas que iniciaram suas operações no ano de 1943 e empresas que iniciaram em 1989. Esse resultado permite verificar a longevidade dessas empresas.

O tipo de layout das indústrias moveleiras da região metropolitana do Rio de Janeiro, em sua maioria, é caracterizado por processo (Figura 3).

A escolha por esse tipo de layout está relacionada à produção de peças que compõe os móveis nessas indústrias. Por se tratar de uma fabricação sob medida ou unitária, há diferenças de materiais, medidas e formatos para cada projeto realizado. Esse arranjo é o mais comum utilizado internamente nas indústrias moveleiras com essa característica. Porém para o acabamento final dos produtos, pode-se dizer que as empresas utilizam layout posicional, visto que peças componentes, máquinas e ferramentas e pessoas são deslocadas para o local de montagem final (residência do cliente) (SANTIN, 2007).

Para cada organização existe um layout específico, o qual é escolhido pela empresa em função de alguns fatores, tais como espaço necessário para cada máquina e seus operadores; tipo de produto fabricado; sequência das operações e número de funcionários envolvidos no processo de fabricação dos produtos (SANTIN, 2007). Uma alteração no layout implicaria em mudanças de outras partes da empresa, principalmente no que diz respeito aos custos (de investimento e de produção), tornando-o trabalhoso e demorado.

\section{Design dos produtos}

As empresas investigadas não apresentaram um departamento específico e estruturado. Seus produtos são elaborados por projetistas por meio das informações oferecidas pelos clientes. A estratégia tradicionalmente aplicada pelo setor é acompanhar a moda internacional e reproduzir nos produtos nacionais as inovações bem-sucedidas oriundas de outros países (ABDI, 2009a).

O desenvolvimento e a incorporação de design próprio aos produtos é um dos maiores desafios competitivos da indústria moveleira nacional. A inovação é o elemento central na criação de maior valor por esta indústria, além de permitir uma inserção ativa no comércio internacional. O design é o único fator de inovação que pode ser próprio ou exclusivo de cada indústria de móveis, já que equipamentos, máquinas e novos materiais podem ser adquiridos por qualquer indústria que queira melhorar seu padrão tecnológico (ARRUDA, 2009).

O Rio de Janeiro tem desenvolvido algumas ações relacionadas ao design, como por exemplo, a “Oficina Rio Design Indústria”, projeto baseado em uma parceria entre indústrias moveleiras das regiões da baixada fluminense e região serrana com designers do Rio de Janeiro; “Giro Design”, ciclo de palestras que leva as tendências internacionais às indústrias de móveis do Rio e do interior do estado; "Rio Design Indústria" que reúne casos de sucessos de design em indústrias e dissemina informações sobre linhas de financiamento para investimento nesse segmento; e por último, o "Fórum de Design" com reuniões regulares entre industriais, designers e acadêmicos para debater e definir estratégias para alavancar o desenvolvimento do estado e da indústria através do design (FIRJAN, 2013a). Cabe ainda ressaltar que o Rio de Janeiro apresenta empresas especializadas em design e desenvolvimento de produtos, o que pode ajudar na melhoria da imagem desse fator no Estado. 


\section{Máquinas e equipamentos}

Quanto às inovações tecnológicas em processo, merecem destaques nas empresas moveleiras da região as progressivas substituições de máquinas e equipamentos mecânicos por tecnologias informatizadas de controle numérico computadorizado (CNC). Processos que eram realizados manualmente, hoje, são executados por máquinas.

As máquinas utilizadas na produção das indústrias moveleiras da região são de origem da Itália e da Alemanha. No ramo de máquinas para madeira, o Brasil é predominantemente um importador (HOFMANN et al., 2007).

As fabricantes de máquinas para madeira constituem-se em um segmento da indústria de bens de capital ainda pouco estudado, diferente de outros setores como a indústria de veículos automotores, equipamentos de telecomunicações ou equipamentos de informática, nos quais existe um dinamismo tecnológico mais evidente (HOFMANN et al., 2007).
Uma possibilidade para aquisição de equipamentos e maquinários no setor moveleiro seria a abertura de uma linha de crédito, o que permitiria a obtenção de capital de giro pelas empresas moveleiras, concedendo as mesmas mudanças na estrutura produtiva (SANT ANNA, 2013).

\section{Produção}

O fluxograma de produção detectado é apresentado na Figura 4.

O fluxo de produção dessas empresas assemelha-se com o encontrado por Kozak et al. (2008), onde a sequência do processo de fabricação de móveis sob encomenda é composta das etapas de corte, aplicação da fita de borda, furação, montagem e produto.

O IBGE (Instituto Brasileiro de Geografia e Estatística) classifica a indústria de móveis pelas matérias-primas predominantes: madeira, metal e plástico (SELLITTO et al., 2014).

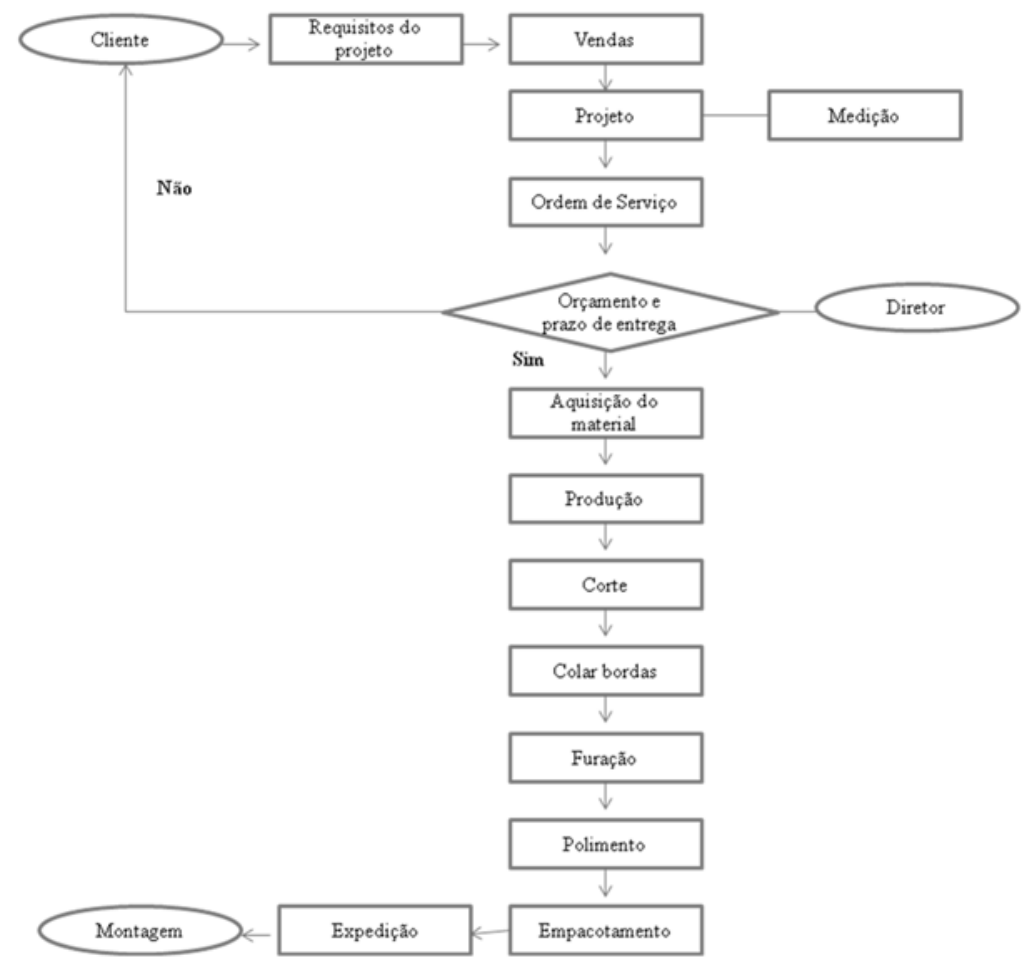

Figura 4. Fluxograma de produção das empresas moveleiras.

Figure 4. Flow Diagram of production of furniture companies. 
As indústrias moveleiras pesquisadas utilizam como principal matéria-prima a madeira e seus derivados. Este resultado coincide com os dados da FIRJAN (2012), onde as unidades fabris do segmento moveleiro do estado do Rio de Janeiro produzem móveis a partir de painéis de madeira industrializada, painéis compensados, madeira serrada ou ainda madeira in natura.
As indústrias de móveis também podem ser segmentadas por categoria de uso: residenciais, escritório e institucionais (utilizados em escolas, lazer, hospitais, restaurantes, hotéis e similares) (PEREIRA, 2009). As respostas obtidas pelos questionários permitem verificar que a maioria das indústrias moveleiras da região metropolitana do Rio de Janeiro pertence ao grupo do mobiliário residencial (Figura 5). Esse resultado

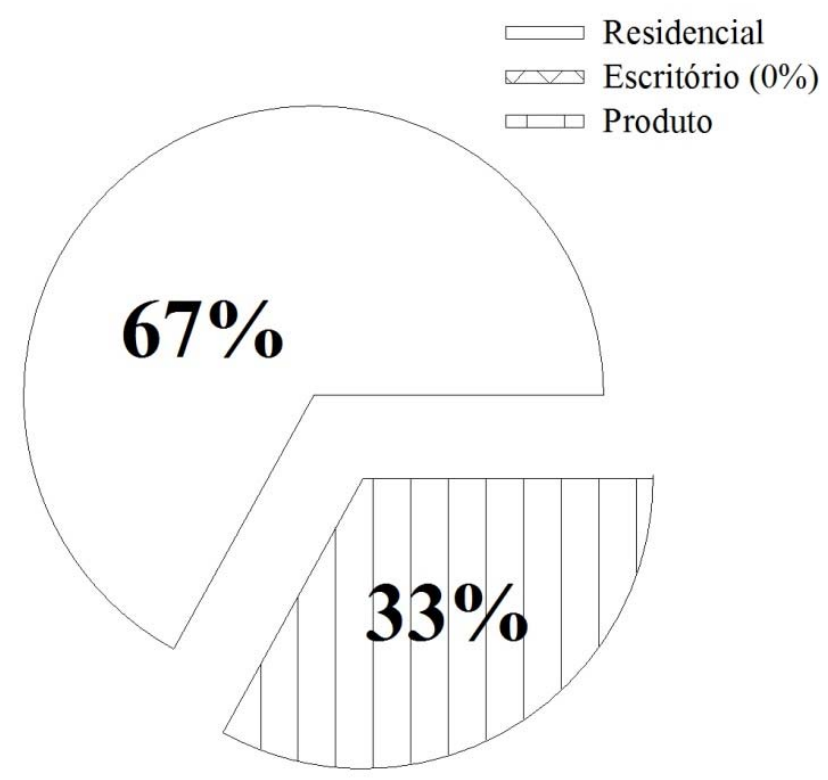

Figura 5. Classificação das indústrias moveleiras da região metropolitana do Rio de Janeiro quanto à categoria de uso.

Figure 5. Classification of furniture companies in the metropolitan region of Rio de Janeiro according to their category.

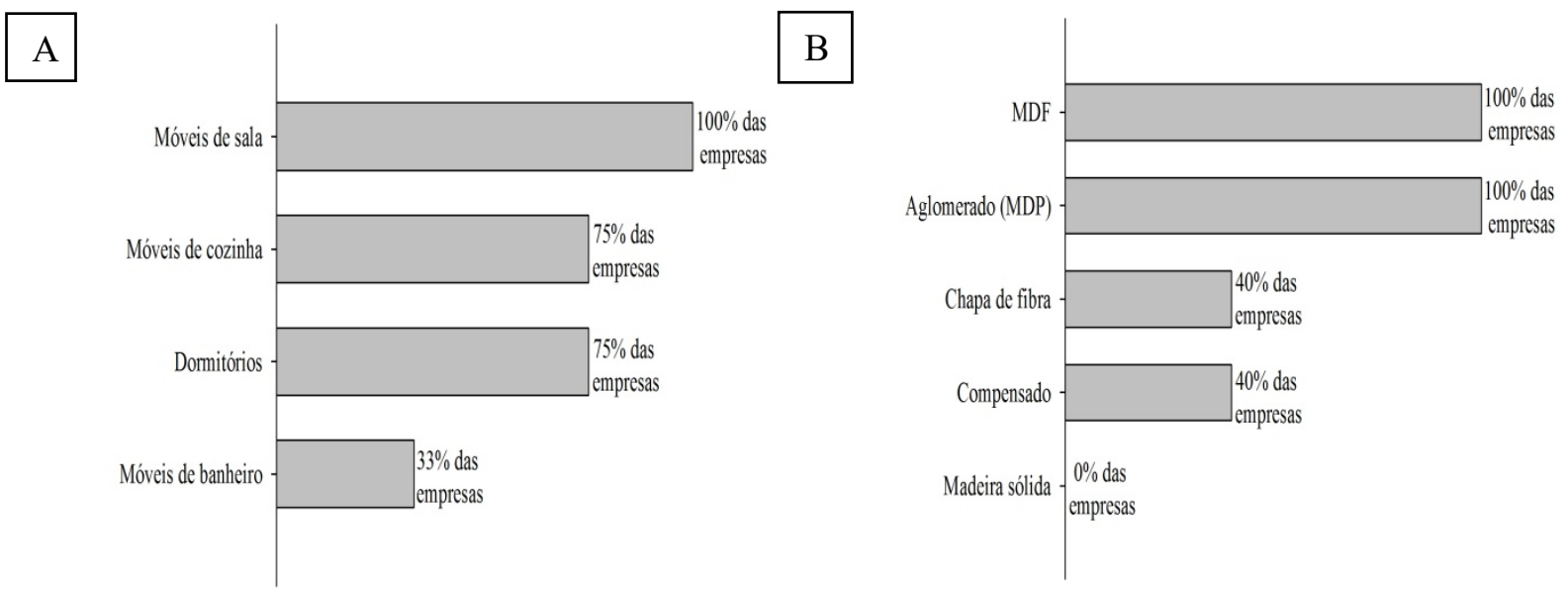

Figura 6. Principais produtos fabricados pelas empresas moveleiras (A) e matérias-primas utilizadas na fabricação dos produtos (B).

Figure 6. Main products manufactured by the furniture companies (A) and raw materials used in the manufacturing of the products (B). 
coincide com os dados apresentados para o setor moveleiro nacional, onde aproximadamente $70 \%$ do total produzido de móveis são residenciais (ABDI, 2009a).

Para Parapinski (2012), o mobiliário residencial se divide em móveis para uso em dormitórios, móveis para uso em cozinhas e outros móveis (sala de estar, sala de jantar e móveis para uso de aparelhos eletrônicos). Avaliando os tipos de móveis produzidos pelas indústrias moveleiras da região metropolitana do Rio de Janeiro, pode-se verificar que todas as empresas da categoria residencial produzem móveis de sala (100\%), 75\% fabricam móveis de cozinha e dormitórios, e $33 \%$ trabalham com móveis de banheiro (Figura 6A).

Quanto às matérias-primas utilizadas pelas indústrias para fabricação desses produtos, constatou-se que todas as empresas empregam o MDP (Medium Density Particle board) (100\%) e o MDF (Medium Density Fiber board) (100\%), 40\% delas utilizam chapa de fibra e compensado e nenhuma indústria processa madeira sólida (in natura) (Figura 6B).

Ambros (2011) destaca que, em relação à linha de produtos, os principais polos moveleiros nacionais produzem móveis residenciais, com destaque para a fabricação de armários, racks e dormitórios. As principais matérias-primas utilizadas na indústria moveleira são as chapas de madeira processada (aglomerado, MDP e MDF) e a madeira maciça proveniente de florestas plantadas. Do total de madeiras consumidas na fabricação de móveis no Brasil, apenas 7\% correspondeu a madeiras maciças, $36 \%$ a madeiras reflorestadas (pinus e eucalipto) e 57\% a painéis de madeira (GALINARI et al., 2013).

A faixa de consumo mensal de painéis (aglomerado, compensado, MDF, chapa de fibra) nas indústrias moveleiras da região metropolitana do Rio de Janeiro compreende está entre 90 e $120 \mathrm{~m}^{3}$ mês $^{-1}$. Esse consumo pode ser considerado baixo, visto que o polo moveleiro de Ubá-MG apresentou um consumo mensal médio de painéis (aglomerado, compensado, MDF e laminado) em torno de $3226 \mathrm{~m}^{3}$ mês $^{-1}$ em 2002 (OLIVEIRA et al., 2010). Comparando-se os consumos, pode-se verificar a distância da produção entre as indústrias do Rio de Janeiro e as indústrias de Ubá, considerada uma das principais regiões produtora de móveis.

Vários fatores afetam o desenvolvimento do mobiliário da região metropolitana do Rio de Janeiro. Foi possível determinar que os empresários moveleiros da região consideraram a escassez de mão-de-obra qualificada (100\%) o fator limitante para a ampliação do setor, superando até mesmo os investimentos gerados pela aquisição de máquinas e equipamentos (40\%) (Figura 7).

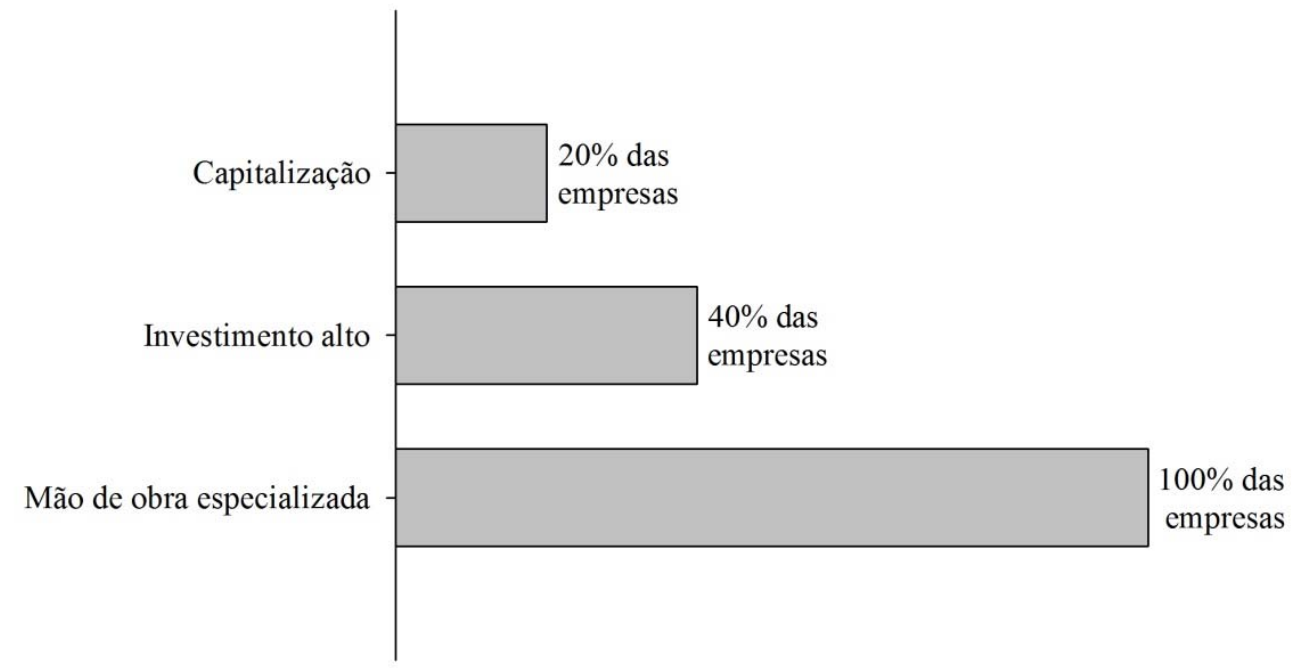

Figura7. Fatores que afetam o desenvolvimento das empresas moveleiras.

Figure 7. Factors that affect the development of the furniture companies. 
A capacitação da força de trabalho afeta o aproveitamento da matéria-prima e a eficácia da produção, pois os funcionários são os responsáveis por grande parte das decisões de ação que determinam o rendimento da fabricação, tais como a escolha de peças a serem processadas para um determinado componente/produto e o plano de corte.

\section{Mercado}

O destino da produção das empresas moveleiras da região metropolitana do Rio de Janeiro é voltado exclusivamente para o mercado interno. A distribuição é concentrada na região sudeste. De acordo com Saraiva (2014), esta região é o principal polo consumidor de móveis do Brasil (R\$23 bilhões).

As indústrias moveleiras da região metropolitana do Rio de Janeiro não participam do mercado internacional e não demonstram interesse em melhorar tal situação, visto que nenhuma atividade é desenvolvida sobre esse tema na região, como exemplo, consórcios com ênfase na exportação.

As indústrias moveleiras da região atendem apenas a demanda de móveis dos consumidores pertencentes à classe A. Em relação ao desempenho das empresas no mercado de móveis, constatou-se que $60 \%$ consideraram à concorrência e o preço da matéria-prima os principais empecilhos para atuarem no comércio moveleiro (Figura 8A). Quanto ao cenário ideal para atuação das empresas moveleiras, verificou-se que $80 \%$ das indústrias apontaram à redução de impostos como fator primordial e $60 \%$ consideraram a capacitação e disponibilidade de mão de obra (Figura 8B).

A concorrência refere-se ao lançamento de novos modelos de móveis que são rapidamente copiados por outras empresas. Um dos maiores problemas enfrentados pelo setor diz respeito à presença de empresas informais. Pelo fato de não recolherem os impostos, estas empresas podem proporcionar orçamentos mais vantajosos aos clientes (AMBROS, 2011). O alto preço da matéria-prima (painéis de madeira, ferragens, tintas, vernizes e colas) está relacionado com a ausência da produção destes insumos no Rio de Janeiro, acarretando em um comprometimento de aproximadamente $30 \%$ dos custos industriais (TAMMELA; CANEN, 2005).

$\mathrm{Na}$ tentativa de reverter o quadro de carência de mão-deobra qualificada, os sindicatos das indústrias moveleiras do Rio de Janeiro, Sincocimo e Sim-Rio, realizam com frequência, cursos, palestras, seminários, consultorias e outras ações em parceria com o SEBRAE e SENAI para aperfeiçoar e valorizar o desenvolvimento das empresas (FIRJAN, 2013b). Os empresários desconhecem as possíveis vantagens das certificações, fato confirmado pela ausência de qualquer tipo de certificado nas empresas.

Os consumidores da região metropolitana do Rio de Janeiro não se preocupam com a existência de certificados de qualidade no ato da compra. Esse fato contribui para a inexistência de busca pelos selos de qualidade. Essa situação somente será revertida quando, possivelmente, os consumidores se tornarem conscientes em relação ao tema. O mercado externo já apresenta compradores com esse perfil, representando nichos de mercados alcançados (ALVES et al., 2009).

A divulgação é a forma como o empreendedor informa ao mercado sobre seu produto, sua marca ou qualquer outro assunto relacionado ao seu negócio. Quanto aos principais meios utilizados pelas empresas moveleiras na divulgação dos produtos, constatou-se que $80 \%$ das indústrias usam os folhetos e os sítios da internet (Figura 9).

A propaganda em meios impressos (catálogos, jornais, revistas, folders e encartes), eletrônicos (televisão e rádio) e virtuais (sítios das fábricas e lojas) tem forte abrangência na comercialização de móveis (MENGATTO, 2012). Entre os diferentes tipos de publicação, o folheto é o instrumento de destaque entre os compradores, visto que torna visível a imagem do produto. A divulgação dos produtos moveleiros por meio 


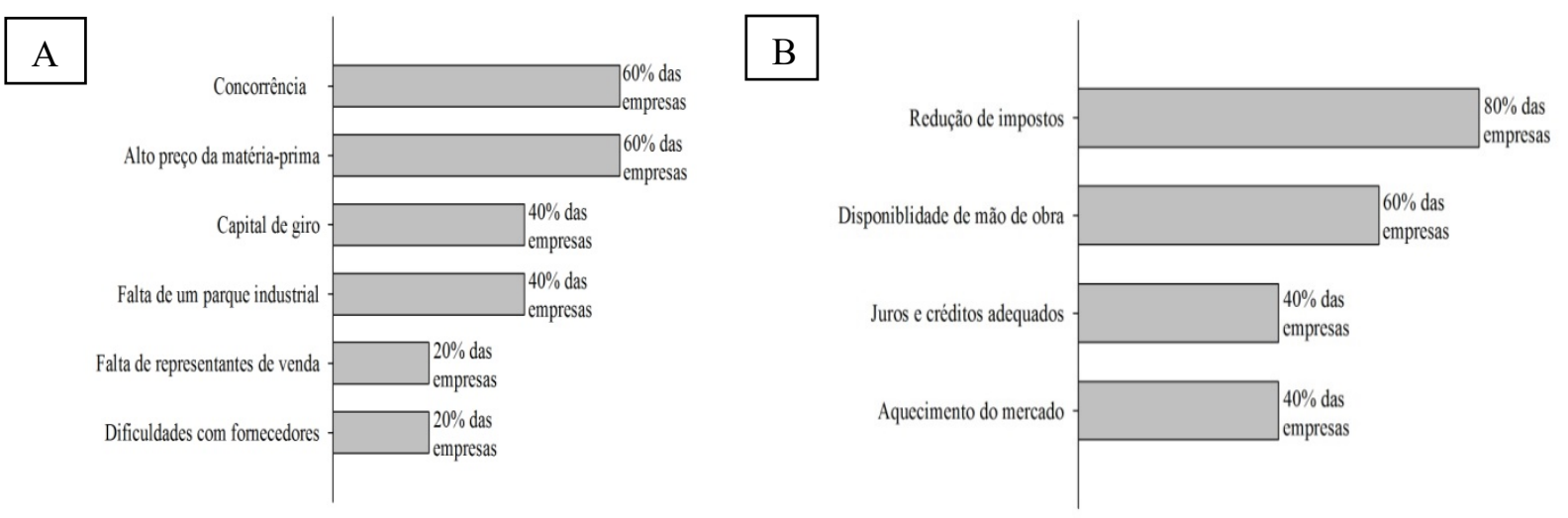

Figura 8. Fatores que afetam a atuação das empresas moveleiras no mercado de móveis (A) e cenário ideal para atuação (B). Figure 8. Factors that affect the performance of the furniture companies in the mobile market (A) and ideal scenario for operation (B).

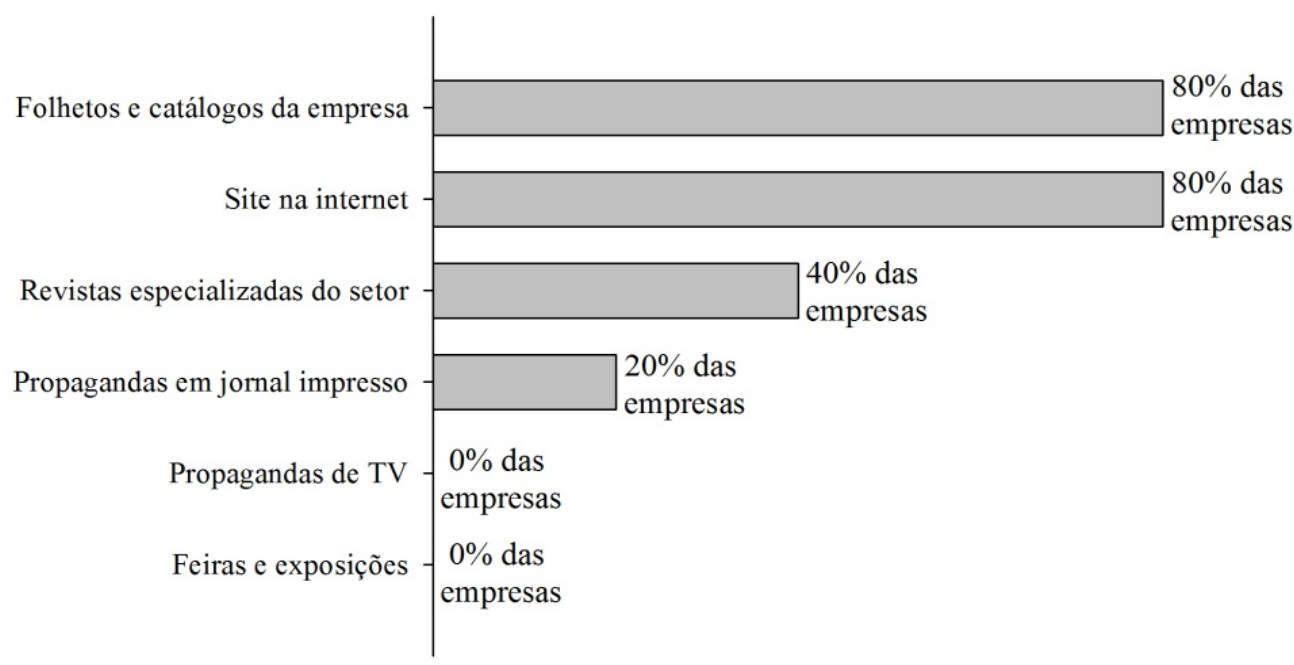

Figura 9. Meios utilizados pelas indústrias moveleiras para divulgar os seus produtos.

Figure 9. Procedures used by furniture companies to advertise their products.

por meio de sítios da internet proporciona às empresas um novo e poderoso canal de comunicação com o mercado.

\section{Perfil Ambiental}

As questões ambientais fazem parte dos planos estratégicos das empresas moveleiras da região metropolitana do Rio de Janeiro, visto que as mesmas já estão implementando programas ambientais (60\%), ou seja, não atendem apenas à legislação (Figura 10).
As indústrias moveleiras da região destacam como atividades ambientais o envio de resíduos para reciclagem, o desenvolvimento de ações internas de reciclagem dos resíduos industriais, o consumo de menos recursos na produção dos produtos e o uso de matéria-prima oriunda de reflorestamentos.

As principais dificuldades assinaladas por algumas empresas na implantação de conceitos ambientais são a ausência de matéria-prima menos impactantes ao meio ambiente, treinamento, não valorização produtos ambientalmente diferen- 
ciados (conscientização) por parte dos clientes, destino apropriado dos resíduos, adesão das empresas para desenvolver programas comuns a elas, custos gerados pela implementação de programas ambientais, falta de fornecedores capacitados ambientalmente, desenvolvimento de produtos em conjunto com os fornecedores, incentivos às empresas fabricantes e a utilização dos resíduos como matéria-prima para outros produtos (ARGENTA, 2007).

Estima-se que menos de 5\% das empresas estão adequadas e praticam algum plano de conservação ambiental, ou seja, prevenção dos impactos causados pelo seu processo de fabricação (ABDI, 2009b). É preciso promover a conscientização da importância da adoção dos requisitos ambientais e incentivar os seus colaboradores para a busca de fornecimento de bens e serviços sustentáveis.

\section{Gestão social}

Foi identificado que os funcionários das empresas moveleiras utilizam equipamentos de proteção individual (EPI). Esse resultado é positivo, já que em micro e pequenas empresas as necessidades dos trabalhadores, no que tange ao respeito à saúde e segurança do trabalho, não são atendidas adequadamente (DEMAJOROVIC; SILVA, 2010).

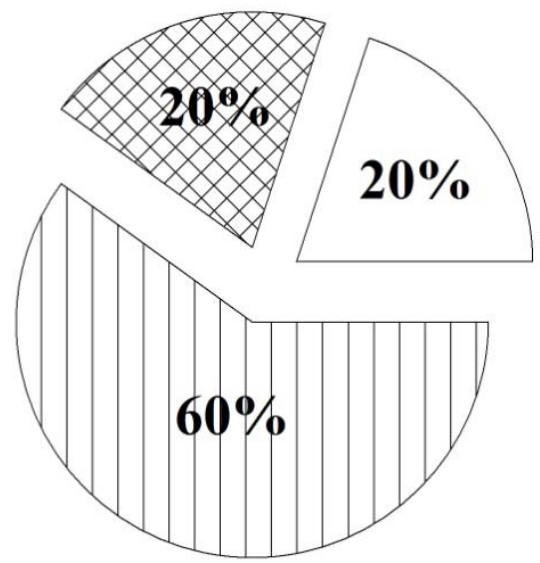

Os principais EPI detectados nas indústrias moveleiras da região metropolitana do Rio de Janeiro foram óculos de segurança, luvas, protetor auditivo, uniforme e calçado de segurança

De acordo com Oliveira (2012), cerca de 2,2 milhões de pessoas morrem anualmente em decorrência de acidentes e doenças de origem profissional. Tais fatalidades podem-se relacionar ao não cumprimento das normas e procedimentos, a não utilização de EPI, a imprudência pessoal e a terceirização de serviços.

Nas empresas que processam a madeira como matériaprima, várias partículas estão suspensas no ar e o ambiente é rico em substâncias químicas e partículas orgânicas (SILVANI et al., 2013), as quais podem provocar riscos à saúde do trabalhador.

Além do risco de acidentes com máquinas e equipamentos, os trabalhadores da indústria moveleira estão expostos a riscos químicos (colas, solventes, tintas, vernizes, resina), riscos físicos (vibração, ruído, umidade, temperaturas elevadas); risco ao manuseio inadequado das ferramentas, riscos ergonômicas relacionados ao manuseio de peças grandes e pesadas, iluminação inadequada, riscos biológicos relacionados a exposição à bactérias e fungos que podem estar presentes na madeira bruta e que causam micoses (MUKAI, 2012).

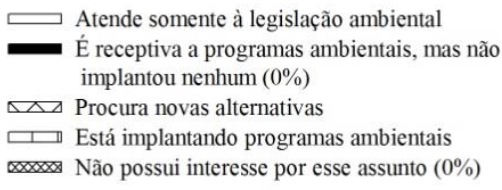

Figura 10. Posicionamento das empresas moveleiras da região metropolitana do Rio de Janeiro com relação às questões ambientais.

Figure 10. Positioning of the furniture companies in the metropolitan region of Rio de Janeiro for the environmental issues. 
A questão da saúde e segurança do trabalhador é uma preocupação atual nas indústrias moveleiras do Brasil (FERREIRA et al., 2011). O setor responde por elevada frequência de acidentes, com ênfase para mutilações de mãos e dedos. A Previdência Social relatou que nos últimos três anos, nas indústrias moveleiras do país, ocorreram aproximadamente 19 mil acidentes de trabalho, que proporcionaram a invalidez permanente de cerca de 500 trabalhadores (BATISTA et al., 2011).

O setor moveleiro, entre os anos de 2007 e 2009, ocupou a sétima posição em acidentes de trabalhos em relação aos outros setores da indústria. Isso significa uma taxa de incidência de acidentes de trabalhos de 22 ocorrências a cada 1.000 trabalhadores. Já em relação à taxa de letalidade, o setor moveleiro ocupou a $14^{\text {a }}$ posição (SESI, 2010).

A empresa que empregar um número superior a vinte funcionários precisa estabelecer a CIPA (Comissão Interna de Prevenção de Acidentes) para gestão de ações relativas à prevenção de acidentes e doenças relacionadas ao trabalho, buscando conciliar a tarefa com a preservação da vida e a promoção da saúde de todos os trabalhadores (SEGURANÇA E MEDICINA DO TRABALHO, 2008).

A maioria das empresas moveleiras da região possui funcionários treinados de acordo com as exigências da CIPA. A criação da CIPA permite promover a melhoria das condições dos ambientes de trabalho por meio da ação dos próprios trabalhadores, visto que a comissão é composta por representantes do empregador e dos empregados (MUKAI, 2012).

\section{Conclusões}

A produção moveleira da região pode ser dividida em duas classes, sendo uma representada pela maioria dos produtores moveleiros (70\%), destacando pequenos estabeleci- mentos dedicados à fabricação de móveis, com poucas máquinas, sendo a produção conduzida por encomenda e ausência de controle. A segunda classe é representada por fábricas modernas contendo parque industrial, de pequeno porte, familiares e de capital nacional.

A produção moveleira da região metropolitana do Rio de Janeiro carece de incentivos e apoio, visto que a maioria dos empreendimentos dedicados à fabricação de móveis ainda é representada por pequenos marceneiros.

O presente trabalho poderá servir de base para os órgãos que estudam o setor moveleiro, visto que, foi possível levantar os principais pontos positivos e negativos das indústrias de móveis da região metropolitana do Rio de Janeiro.

\section{References}

ABDI - Agência Brasileira de desenvolvimento Industrial, Centro de Gestão e Estudos Estratégicos. Estudo Prospectivo Madeira e Móveis. Brasília: Agência Brasileira de desenvolvimento Industrial, 2009a. 210p.

ABDI - Agência Brasileira de desenvolvimento Industrial, Centro de Gestão e Estudos Estratégicos. Panorama Setorial Madeira e Móveis. Brasília: Série Cadernos da indústria ABDI IX, 2009b. 208p.

ALVES, R. R.; JACOVINE, A. G.; SILVA, M. L.; VALVERDE, S.; R.; SILVA, J.; C.; NERDELLI, A. M. B. Certificação florestal e o mercado moveleiro nacional. Árvore, v. 33, p. 583-589, 2009.

AMBROS, J. D. Cadeia produtiva moveleira da região central do estado de Tocantins: caracterização e perspectivas para a formação de um pólo moveleiro. Tese (Doutorado em Ciência Florestal). Universidade de Brasília, Distrito Federal, DF, 2011. 301p.

ARGENTA, D. O. F. Alternativas de melhoria no processo produtivo do setor moveleiro de Santa Maria/RS: impactos ambientais. Dissertação (Mestrado em Engenharia de Produção). Universidade Federal de Santa Maria, Santa Maria, RS, 2007. 122p. 
ARRUDA, G. L. R. C. O Design na Indústria Moveleira Brasileira e seus Aspectos Sustentáveis: estudo de caso no polo moveleiro de Arapongas-PR. Dissertação (Mestrado em Design). Faculdade de Arquitetura, Artes e Comunicação da Universidade Estadual Paulista Júlio de Mesquita Filho. São Paulo, SP, 2009. 120p.

BATISTA, J. H. L.; RODRIGUES, G. L.; MOREIRA, M. C. Fundacentro desenvolve projeto para levantar debate das relações entre saúde e trabalho no polo moveleiro. Disponível em: < http://www.madeiratotal.com.br/noticia.php?id=9761\&volta $=$ noticias.php? cat $=24>$.

2011. Acesso em: 24 mar. 2015.

BOA, A. C.; DONATELLI, J. S.; ANDRADE, W. S. P.; NOGUEIRA, M. A. Análise do Layout de uma Indústria Moveleira Localizada no Polo de Linhares. Floresta e Ambiente, v. 19, n. 3, p.155-161, 2012.

CASSILHA, A.; C. PODLASEK, C. L.; JUNIOR, E. F.C.; SILVA, M.C.; MENGATTO, S. N. F. Indústria moveleira e resíduos sólidos: considerações para o equilíbrio ambiental. Revista Educação \& Tecnologia, n. 8, p. 209-228, 2004.

DEMAJOROVIC, J.; SILVA, A.V. Arranjos produtivos locais e práticas de gestão socioambiental: uma análise do polo moveleiro de Arapongas. Ambiente \& Sociedade, v.13, p. 131$149,2010$.

EXATA COMUNICAÇÃO. Abimóvel apresenta Plano de Trabalho 2014/2015 para o Setor Moveleiro. Disponível em: $<$ http://moveistotal.com.br/noticia.php?id=27407\&volta=index.php $>$

FERREIRA, C. B.; MINETTE, L. J.; SOUZA, A.P.; PEREIRA, R. F.; RODRIGUES, V. A. J.; BRAGA, C. S. Análise das condições de segurança do trabalhador em uma indústria moveleira do estado de Minas Gerais. In: ERGOFLOR, 2011. Viçosa. Anais...Viçosa-MG. 2011.

FIRJAN - Federação das Indústrias do Rio de Janeiro. Estudo de mercado: consumo de produtos de base florestal. Curitiba: FIRJAN, 2012. 32p.

FIRJAN - Federação das Indústrias do Rio de Janeiro. FIRJAN é a instituição de apoio ao design do ano. Disponível em: $\quad<$ http://www.firjan.org.br/data/pages/2C908CEC42014883014229406C6D4148.htm>. 2013a.
FIRJAN - Federação das Indústrias do Rio de Janeiro. Indústria moveleira cria plano para recuperar mercado. Disponível em: <http://www.sim-rio.org.br/artigo/industria-moveleira-cria-plano-para-recuperar-mercado $>$. 2013b.

FIRJAN - Federação das Indústrias do Rio de Janeiro. Rio exporta- Boletim de comércio exterior do Rio de Janeiro. Rio de Janeiro: Firjan, 2010. 18p.

GALINARI, R.; JUNIOR, J. R. T.; MARGADO, R. R. A competitividade da indústria de móveis do Brasil: situação atual e perspectivas. BNDES, n. 37, p. 227-272, 2013.

GALLUCCI, M. Consumo de móveis cresceu em um ritmo próximo a 7\% ano. Disponível em: <http://www.emobile.com.br/site/varejo/estudo-varejo-iemi/>. 2014. 2015.

HOFMANN, R.; AQUINO, D.; MELO, M.; PELAEZ, V. Perfil da indústria de máquinas para madeira no estado do $\mathrm{Pa}$ raná. In: Encontro de Economia Paranaense, 2007, Curitiba. Anais... Curitiba: UFPR, 2007.

KOZAK, P.A.; CORTEZ, A. M.; SCHIRMER, W. N.; CALDEIRA, M. V. W.; BALBINOT, R. Identificação, quantificação e classificação dos resíduos sólidos de uma fábrica de móveis. Revista Acadêmica de Ciências Agrárias e Ambientais, v. 6, p. 203-212, 2008.

MATTIA, A. A.; TONI, D.; LARENTIS, F.; FINATTO, C. Perfil da Indústria Moveleira do Rio Grande do Sul. Bento Gonçalves: NUPARVI, 2007. 153p.

MENGATTO, S. N. F. Critérios para o design de estação de trabalho informatizada residencial. Tese (Doutorado em Design e Arquitetura). Faculdade de Arquitetura e Urbanismo de São Paulo, São Paulo, SP, 2012. 2012p.

MUKAI, H. Sistemas integrados de gestão de design, qualidade, ambiente, saúde e segurança no trabalho: aplicação às pequenas e médias empresas do setor moveleiro. Tese (Doutorado em Engenharia de Produção). Universidade Federal de Santa Catarina, Florianópolis, SC, 2012. 277p.

OLIVEIRA, C. A. D. Segurança e Saúde no Trabalho - Guia de Prevenção de Riscos. São Caetano do Sul: Yendis. 2012. $161 \mathrm{p}$.

OLIVEIRA, P. R. S.; VALVERDE, A. E. L.; MENDONÇA, F. M.; ALVARENGA, A. P. V. VALVERDE, S. R.; MARQUES, G. M. Cadeia produtiva da movelaria: polo moveleiro de Ubá. Série Documentos EPAMIG, 2010. 66p. 
PARAPINSKI, M. L. Desempenho das exportações brasileiras de móveis de madeira - 1991 a 2010. Dissertação (Mestre em Ciências Florestais). Universidade Federal do Paraná, Paraná, PR, 2012. 154p.

PEREIRA, D. Competências da gestão estratégica do design no polo moveleiro do Alto Vale do Rio Negro (SC). Dissertação (Mestrado em Engenharia de Produção). Universidade Tecnológica Federal do Paraná. Paraná, PR, 2009.115p.

ROSA, S. E. E.; CORREA, A. R.; LEMOS, M. L. F.; BARROSO, D. V. O setor de móveis na atualidade: uma análise preliminar. BNDES, n. 25, p. 65-106, 2007.

SANT ANNA, L. A. N. Arranjos Produtivos Locais Potenciais: Um Estudo sobre o Setor Moveleiro no Município de Duque de Caxias. Dissertação (Mestrado em Administração). Universidade do Grande Rio "Prof. José de Souza", Duque de Caxias, RJ, 2013. 93p.

SANTIN, A. Tipos de layout e sua aplicação na indústria moveleira. Rio Grande do Sul: SENAI/CETEMO, 2007. 22p.

SEBRAE - Serviço de Apoio às Micro e Pequenas Empresas. Anuário do trabalho na micro e pequena empresa: 2013. Brasília: DIEESE, 2013. 284p.

SEGURANÇA E MEDICINA DO TRABALHO. São Paulo: Atlas, 2008. 797p.

SELLITTTO, M. A.; ROSSATTO, J. P.; LUZZI, I.; PEREIRA, G. M.; BORCHARDT, M. Análise descritiva de fatores que influenciam resultados econômicos no cluster moveleiro de Bento Gonçalves. Revista Produção Online, v.14, p. 11921215, 2014.

SESI. Panorama em Segurança e Saúde do Trabalho (SST) na indústria: Brasil e Unidades da Federação 2007. Brasília: SESI/DN, 2010. 2p.

SILVANI. I. O. F. M.; GRASEL, E.; PANSERA, S. Perfil respiratório dos trabalhadores da indústria moveleira do município de Coronel Freitas-SC. FisiSenectus, n. 1, p. 43-50, 2013.

SIMIOLI, E. R. Aplicação de princípios da gestão e ferramentas da qualidade no polo moveleiro de Votuporanga. Dissertação (Mestrado em Engenharia de Produção). Universidade Paulista. São Paulo, SP, 2010. 136 p.

TAMMELA, I.; CANEN, A. G. A competição baseada no tempo: um estudo de caso na indústria moveleira do Rio de Janeiro. Revista Produção online, v. 5, p.1-5, 2005. 